\title{
Vestibular evoked myogenic potentials: an overview
}

Renato Cal', Fayez Babmad Jr. ${ }^{2}$

Keywords: endolymphatic hydrops, evoked potentials, vestibular.

\section{Summary}

\begin{abstract}
$T_{1}$ he vestibular evoked myogenic potential (VEMP) test is a relatively new diagnostic tool that is in the process of being investigated in patients with specific vestibular disorders. Briefly, the VEMP is a biphasic response elicited by loud clicks or tone bursts recorded from the tonically contracted sternocleidomastoid muscle, being the only resource available to assess the function of the saccule and the lower portion of the vestibular nerve. Aim: In this review, we shall highlight the history, methods, current VEMP status, and discuss its specific application in the diagnosis of the Ménière's Syndrome.
\end{abstract}

\footnotetext{
${ }^{1}$ Otolaryngologist. Researcher at the Department of Otology - Massachusetts Eye and Ear Infirmary, Harvard Medical School, Boston, MA, EUA. ${ }^{2}$ Otolaryngologist Researcher at the Department of Otology - Massachusetts Eye and Ear Infirmary, Harvard Medical School, Boston, MA, EUA. Assistant Physician - Department of Otorhinolaryngology and Head and Neck Surgery - Hospital Universitário da Universidade de Brasília. Graduate Student (PhD0 in Medical Sciences Universidade de Brasília (UnB), Brasília, DF, Brazil.

Harvard Medical School, Boston, MA, EUA. Departamento de Otorrinolaringologia e Cirurgia de Cabeça e Pescoço do Hospital Universitário de Brasília, Brasília, DF, Brazil.

Send correspondence to: Renato Cal - Rua Domingos Marreiros 49 sala 910 Belém PA Brazil 66055-210. E-mail: renato@renatocal.com

The author received a research grant from CNPQ - MEC (PhD Scholarship) - Fayez Bahmad Jr. Paper submitted to the BJORL-SGP (Publishing Management System - Brazilian Journal of Otorhinolaryngology) on March 5, 2007;
} and accepted on March 13, 2007. cod. 3725 


\section{INTRODUCTION}

Vestibular evoked myogenic potentials (VEMP) are inhibitory electrical potentials generated after a sound stimulus (clicks or pure tones), originated in the saccule and conducted by the lower portion of the vestibular nerve all the way to the central nervous system (CNS), generating inhibitory electrical responses picked up by electrodes placed on the sternocleidomastoid muscle (SCM $)^{1,2}$. In English, these potentials are known by the acronym VEMP, meaning Vestibular Evoked Myogenic Potentials. The reason why we have had a growing interest on this topic in recent years is because of the physiological origin (saccule and lower division of the vestibular nerve) of these potentials and their possible clinical applications.

Under the evolutionary viewpoint, the cochlea was the last portion to develop within the membranous labyrinth $^{3-5}$, which suggests that in some species of fish and even in some mammals, the saccule also has an auditory function, causing some authors to speculate about the existence of some sensorial cells to sound stimulus in the saccule of human beings $s^{4,6,7}$. Still considering this evolutionary characteristic, it is believed that when we are submitted to a high intensity sound stimulus, these remaining saccule cells are stimulated, triggering an inhibitory reflex to the ipsilateral SCM muscle, causing its relaxation with consequent contraction of the contralateral muscle.

It is exactly this unilateral inhibitory reflex which we capture with electrodes positioned on the SCM muscle that we know as VEMP. These are exactly the remaining hair cells in the saccule that would originate VEMP, and this stimulus is taken to the CNS through the lower portion of the vestibular nerve (IVN) $)^{2,7-10}$. This makes VEMP a new method that neurotologists have at hand to diagnose and investigate the vestibular system, because until then there was no exclusive way to assess saccule and IVN function.

\section{OBJECTIVE}

The goal of the present review is to highlight the history, modus operandi, current status of the research involving VEMPs, and also to discuss its specific applications in the diagnosis of Ménière's syndrome, vestibular schwannoma, superior semicircular canal dehiscence syndrome, perilympathic fistula, vestibular neuritis and other vestibular lesions, in an attempt to help otolaryngologists and neurologists work with this new diagnostic tool.

\section{MÉTHOD}

Based on a broad literature review - the authors used the MEDLINE database (www.pubmed.com), and on the clinical experience acquired in a reference tertiary hospital for neurotology disorders in the USA, they carried out an updated analysis on VEMPs.

\section{RESULTS}

\section{Background}

The first sound evoked vestibular responses were described by Von Békésy in 1935. He used high intensity sound stimuli (about $134 \mathrm{~dB}$ ) in order to generate head movements towards the sound stimulus7. The explanation for this phenomenon was the proximity between the stapes footplate and the saccule macula hair cells, which would activate the afferent neurons. This proximity between the stapes footplate and the saccule macula has been broadly described in the international literature, by means of macro and microscopic anatomical studies of the temporal bone ${ }^{11}$.

In 1964 we had the first reports on short-latency myogenic electrical evoked potentials ${ }^{12,13}$; however, it was only 7 years later that Towsend et al. noticed that the true origin of these potentials was the saccule ${ }^{14}$. The authors proved it when they found VEMPs present in deaf patients; however, they were absent in patients submitted to vestibular neurectomy. Later, these same authors discovered that VEMP responses were present in patients who had been submitted to ablation of the semicircular canals by streptomycin and in patients, who had benign paroxysmal postural vertigo (BPPV), while these same potentials were absent in patients with Ménière's syndrome ${ }^{14}$.

In order to reinforce the physiological basis of VEMPs, McCue \& Guinan identified some fibers from the inferior division of the vestibular nerve in cats, which responded electrically to sound stimuli above $80 \mathrm{~dB}$ SPL (sound pressure level), which increased their electrical activity as the stimulus intensified ${ }^{15,16}$, confirming the hypothesis that these muscle inhibitory electrical potentials were originated in the saccule and, consequently, had their afferent pathways in the IVN.

Since then, the interest for this topic has been on the rise, with a significant increase in the number of publications in recent years about VEMPs ${ }^{1,7,17-23}$. It was only in 2005 that more than 30 papers were published in English2, and in 2006 this number went up to 33 (source www.pubmed.com). It is believed that in 2007, this number will be even greater (Figure 1).

\section{VEMP test performance}

These potentials are generated after high intensity sound stimuli (clicks or pure tones), of $0.1 \mathrm{msec}$ of duration, at about 140 to $145 \mathrm{~dB}$ SPL (about $90 \mathrm{~dB}$ hearing level), sounded in an ear phone (monaural or bilateral). The patients are put in a chair, seated and instructed to turn their heads to the opposite side of the sound stimulus, in order to contract the contralateral SCM muscle. VEMPs are read by electrodes placed on the patient's SCM (ipsilateral to the sound stimulus), the positive electrode is placed on the upper third of the muscle, while the negative 


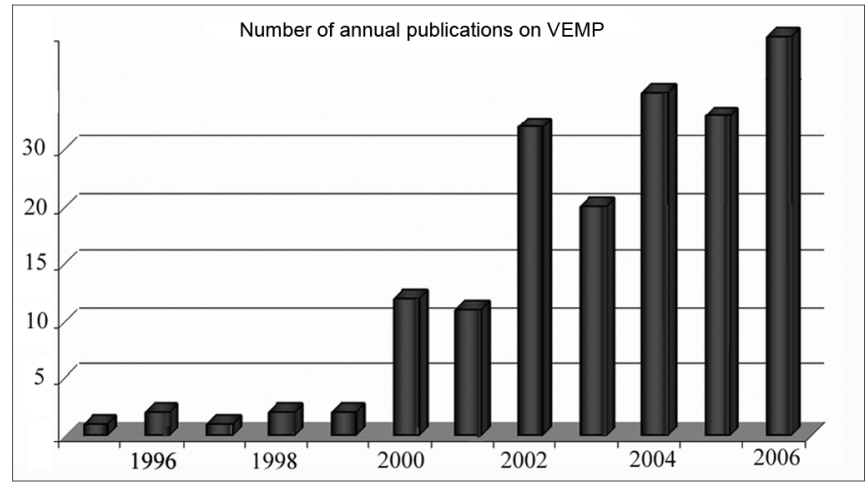

Figure 1. Annual publications on the Vestibular Evoked Myogenic Potential (VEMP) since 1995. Notice that as of the year 2000, there was a quick increase in the number of publications dealing with VEMP use. Source: www.pubmed.com.

electrode is placed on the muscle tendon, just above the clavicle (Figure 2A).

This electrode placement can happen in an inverse way, causing response wave inversion. The ground electrode is usually positioned on the patient's vertex. Although the SCM muscle is the most frequently site used to obtain vestibular responses, some authors attained these responses from other muscles such as the trapezius and muscles in the legs and arms ${ }^{3,24}$.

It is worth to bear in mind that one needs a normal middle ear in order to be able to record VEMPs, since small air-bone gaps of $8.75 \mathrm{~dB}$ are enough to dampen the responses ${ }^{25}$. The electrical responses from these potentials are made up of two biphasic waves, the first is positive,

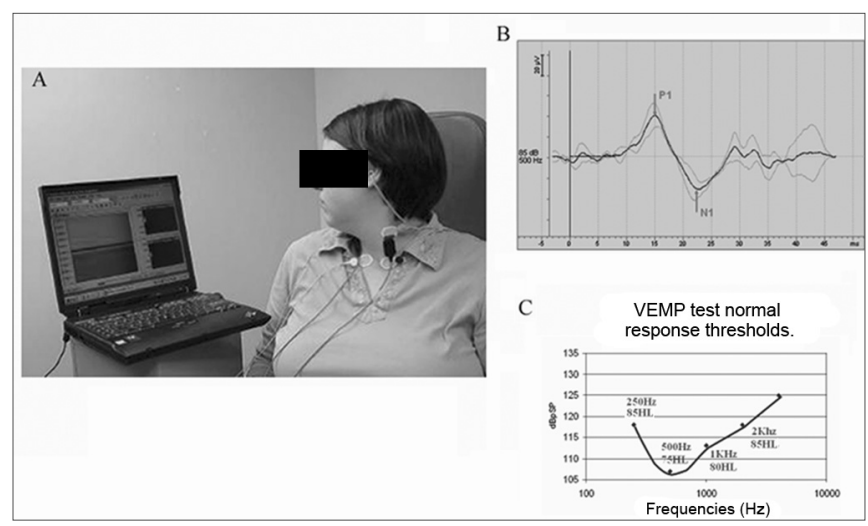

Figure 2a. VEMP test on the left side with earbud phones and electrodes placed on the SCM. The patients are placed on a comfortable chair and are instructed to turn their heads to the direction contrary to that of the sound stimulus, so as to have SCM muscle contraction to the ipsilateral side of the sound stimulus.

Figure 2b. VEMP typical wave in a normal patient. Showing the first positive wave (P1) around $13 \mathrm{~ms}$ and the second negative wave (N1) around 23ms.

Figure 2c. Typical VEMP curve in a normal patient, showing the response threshold of the main frequencies tested. with a latency around 13ms, known as p13; followed by another wave, this time negative, with a latency around $23 \mathrm{~ms}$, known as n23 (Figure 2B).

These responses are present in most of the normal individuals studied ${ }^{5}$, differently from a second biphasic complex known as n34-p44, which according to Colebatch would be absent in $40 \%$ of the normal individuals studied ${ }^{26}$, while Robertson described that this second complex would be present in $68 \%$ of normal individuals 27 . Because of the lack of reproducibility of this second complex (n34-p44), the vast majority of these studies with VEMP only considers the first biphasic complex (p13-n23) 1,2,7-10,17,26,28.

One of the most controversial points regarding VEMPs is the relationship observed between the electrical response amplitudes and the level of contraction of the muscles tested (usually the SCM). This relationship was noticed by Colebatch et al., in 1994 and confirmed by many

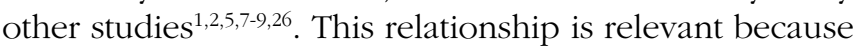
we would need standardized muscle contractions among the patients, and if it does not happen, it becomes very hard to be able to compare the test of an elderly patient with that from a young athlete, who very likely has a much higher level of muscle contraction. Thus, many studies are currently used to monitor SCM contraction, such as electromyography and biofeedback ${ }^{5,7,23}$.

Another controversial point regarding VEMPs is that the sound stimulus is generated through clicks or tone bursts. Many authors study this topic ${ }^{9,10,29}$. In 2003, Cheng et al. did a study in which they compared the influence of clicks and tone bursts in the generation of vestibular responses. They concluded that VEMPs generated by clicks have a greater response index in normal individuals, greater amplitude of waves p13-n23 and lower latency ${ }^{9,29}$. These data show that when the physician is reading a VEMP test, he/she must know which was the sound stimulus used, since it impacts directly on wave amplitude and latency. Nonetheless, in 2004 Rauch et al. showed that within the range of frequencies tested to generate VEMP responses, $500 \mathrm{~Hz}$ was the most sensitive. Moreover, he introduced a new way of analyzing VEMP results, by studying not only amplitude and latency, but also the response threshold in four different frequencies $(250,500,750 \text { and } 1000 \mathrm{~Hz})^{30}$. (Figure 2C)

So far, there are no specific devices to be used in VEMP testing, and most of the Centers use the ABR equipment (audiometric analysis of the auditory nerve and brainstem) to record these responses. Some authors also use other means to trigger these potentials in patients with conductive hearing loss, they use a bone stimulus by gently tapping the skull of the patients with a small hammer ${ }^{31}$. They attained responses in $10 \mathrm{~ms}$ (positive wave) and $17 \mathrm{~ms}$ (negative wave) in patients in whom the sound stimulus was incapable of generating such responses because of their conductive hearing loss. 


\section{Clinical applicability}

Differently from what happens in the auditory system, the vestibular system is much more complex and much less known and studied, that is why the interest on VEMPs has grown in recent years, because these potentials reflect the function of the otolithic organs (saccule) and the IVN, something that so far was not possible to be evaluated by the numerous other vestibular tests, such as vector-electronystagmography, the rotating chair test and the platform test ${ }^{2}$.

The caloric test which, without any doubt is the most used test to check vestibular function, is limited to the workings of the lateral semicircular canal and the upper portion of the vestibular nerve in each side separately, while the platform test does not assess each one of the labyrinths separately, nor one of the systems in charge of body balance (vision, labyrinth and proprioception), separately.

Many publications have shown VEMPs used as a means to diagnose or even to help diagnose the most diverse neurotological diseases, such as Ménière's disease, superior semicircular canal dehiscence, vestibular neuronitis, vestibular schwannomas, control after intratympanic administration of gentamicin and even perilymphatic fistulas $1,2,5,7,19,28,30,32,33$. The role of VEMPs in each one of these situations is described as follows:

\section{Vestibular Neuronitis}

It is one of the most frequent causes of vertigo and it is normally diagnosed based on the patient's clinical history, associated with a caloric test that shows a unilateral functional deficit. 34 According to previously held studies, we know that both branches of the vestibular nerve (inferior and superior) can be affected by this disease; however, based on studies by Goebel et al. in 2001, we can have some explanations about the possible reason why the upper portion is much more frequently affected than its inferior counterpart ${ }^{35}$.

It is exactly based on this differentiation between the upper and lower branches that VEMPs can be utilized. In 1995, Halmagyi et al. studied 22 patients with diagnosis of vestibular neuronitis and did not get caloric responses in the affected sides of these patients. Curiously, he observed that when submitted to VEMP testing, the responses were normal in 6 patients, reduced in 5 and absent in 1133 . This proves that some patients have both portions of the vestibular nerve involved, while others have lesions in its upper portion only. Such fact is very important regarding the prognosis of these patients, since the patients with absent or altered VEMPs will hardly develop BPPV in the posterior semicircular canal, since the nerve that innervates this canal is damaged (IVN). Now, those patients with manifestations of vestibular neuronitis and normal VEMPs show that upper division of the nerve was involved, it is possible that patient will develop posterior canal BPPV in the future ${ }^{36}$.

\section{Superior Semicircular Canal Dehiscence}

In 2001, researchers from the Johns Hopkins University in the United States, headed by Professor Lloyd B. Minor, described a vestibular entity not previously reported in the literature, called superior semicircular canal dehiscence ${ }^{37}$. This syndrome is characterized by vertigo and nystagmus triggered by sound and/or pressure stimuli, and its pathophysiology is based on the "Third Window" theory. The existence of this third window in the internal ear (besides the round and oval windows) would cause an impedance reduction in the inner ear, allowing a better membranous labyrinth fluid movement and consequently a greater deflection of the vestibular sensors to sound and pressure stimuli ${ }^{37-40}$.

Based on this physiopathological explanation, VEMPs have become rather important, since the existence of this third vibratory window would cause a greater movement of the stapes on the oval window, and consequently a greater stimulation on the saccule macula to the sound stimuli employed. This would bring down VEMP thresholds in almost all the frequencies tested, and such data has been confirmed by numerous studies, such as the one carried out by Brantberg et al. in 1999, which tested VEMPs in 3 patients with superior semicircular canal dehiscence, noticing a threshold reduction in the affected side of all patients, especially within the range between $500 \mathrm{~Hz}$ to $1000 \mathrm{~Hz}^{41}$.

A similar study was carried out by this same group in 2001, when 8 patients with superior semicircular canal dehiscence syndrome were submitted to VEMP testing and all had threshold reduction in the affected side; 4 of the 8 patients tested had normal audiometry and 6 of the 8 had normal responses in the caloric test ${ }^{42}$. These studies reinforce the importance of VEMPs in the diagnosis of this syndrome that often times has a varied clinical manifestation, normal audiometric findings or findings that mimic otosclerosis (conductive hearing loss) and that can only be confirmed by means of a high resolution CT-scan ${ }^{37-39,42}$.

\section{Vestibular Schwannoma}

This benign tumor which affects the vestibular pathways is always a diagnostic hypothesis considered in the most varied clinical cases. Currently, the most efficient method to diagnose it, and also the most expensive one is Magnetic Resonance Imaging (MRI). Nonetheless, because of its high cost and the impossibility of performing the test in all suspected patients, the ABR also plays an important role in the diagnosis of vestibular schwannomas

Knowing that VEMP neural pathways involve the inferior portion of the vestibular nerve, this diagnostic method can also be used to help in the diagnosis of vesti- 
bular schwannomas. Some studies in the literature already show this important contribution of VEMPs, such as the one developed by Murofushi et al. in 1998, who observed $80 \%$ of altered VEMPs in 17 patients with established diagnosis of vestibular schwannoma ${ }^{43}$. In 2001, Takeichi et al. performed a similar study in which he observed altered VEMPs in 13 of 18 patients diagnosed with vestibular schwannoma confirmed by MRI ${ }^{44}$.

In a general way, VEMPs can contribute to the diagnosis of vestibular tumors, but must not be used as the sole diagnostic method, because it only assesses the function of the inferior vestibular nerve. Nonetheless, when performed together with MRI, the ABR, audiometry and caloric test, they may help in the exact location of the tumor in the vestibular pathways.

\section{Ménière's Syndrome (MS)}

Among all the clinical applications of VEMPs, this can be the one with the greatest clinical relevance. Besides prior studies in temporal bones, the relationship between the endolymphatic hydrops and MS is well established ${ }^{45-50}$. Schuknecht et al. showed that the most frequently involved sites by endolymphatic hydrops are the cochlea, followed by the saccule and the utriculus, respectively ${ }^{11,46,47,49,51}$. Since the saccule is considered the site of origin of VEMPs, it is expected to find VEMPs altered even in early stages of the Ménière's Syndrome.

In 2004, Rauch et al. published a study in which they showed VEMPs present in about 94\% of the patients with MS in the affected side and the frequency thresholds between $250 \mathrm{~Hz}$ and 2,000 Hz were increased ${ }^{30}$. Nonetheless, one of the most interesting pieces of data of this study was the fact that about $27 \%$ of the asymptomatic ears from these patients with unilateral MS had alterations in this test. When we compared this result to data from temporal bone studies that found about 38\% of endolymphatic hydrops in asymptomatic ears of patients with MS1 and epidemiological studies that reported bilateral involvement of MS in $30 \%$ to $35 \%$ of the patients, we concluded that VEMPs may be a diagnostic method for endolymphatic hydrops in initial stages, and it can serve as a prognostic factor for bilateral involvement in MS.

Many other studies were also published in the international literature recently about VEMPs in patients with MS. In 2001, Murofushi et al. reported that $51 \%$ of the patients with MS had no response to the VEMP test ${ }^{52}$, while Waele et al., doing the same study, observed an index of $54 \%$ of response absence in the VEMP test in patients with $\mathrm{MS}^{53}$. Other authors, performed studies comparing the relationship between the glycerol test and VEMPs, concluding that VEMP results after administering glycerol were altered, and VEMP testing is the only useful method to diagnose endolymphatic hydrops ${ }^{54,55}$.

\section{Perilymphatic Fissure}

In 2006, Modugno et al. from the University of Bologna, Italy, published a study in which VEMP testing was used for the diagnosis of endolymphatic fistula ca$\mathrm{ses}^{56}$. They reported four cases in which VEMP response thresholds were reduced with stimuli in the frequency of $500 \mathrm{~Hz}$. The possible explanation for this threshold lowering is based on the same theory that explains this phenomenon in cases of superior semicircular canal dehiscence, a third vibratory window in the internal ear would reduce the impedance, consequently causing a reduction in these thresholds. One important criticism to this study published in 2006 is the small number of patients tested and the absence of a longer follow up.

\section{Monitoring after treatment with intratympanic gentami- cin}

In recent years, the Ménière's disease treatment in cases resistant to clinical treatment started to be carried out through the administration of intratympanic gentamicin, in an attempt to reduce vertigo symptoms in these patients ${ }^{57}$. After this therapy, many professionals performed an electronystagmography with caloric test in an attempt to confirm if the gentamicin dose employed was enough to cause damage to the vestibular cells and it is exactly with this aim that VEMPs can also be used.

In 2002, De Waele et al., showed that $92 \%$ of the patients submitted to intratympanic injection of gentamicin had no responses in VEMP testing in one month and they kept like that for even one year after treatment ${ }^{58}$.

\section{FINAL REMARKS}

VEMP testing is a new complementary test, which may contribute, together with other neurologic tests, for the diagnosis of many and diverse vestibular disorders, among them we stress: Ménière's Syndrome and Superior Semicircular Canal Dehiscence.

The test is currently used in tertiary reference centers that deal with neurotological disorders. Nonetheless, the authors stress that since this is a new diagnostic method, the use of VEMPs is still not totally adapted to clinical use, since most of the facilities still don't have their own equipment (using the same ABR equipment), trained professionals for their proper realization and interpretation, and lack international standardization to analyze results.

Another extremely important point to be highlighted is the need for standardization in relation to monitoring SCM muscle contraction level, before VEMPs are employed in clinical use, since VEMP responses are broadly dependent on the level of activities of this muscle.

At any rate, VEMP testing seems to be a promising complementary test, especially for providing information on the function of the saccule and inferior portion of the vestibular nerve. 


\section{REFERENCES}

1. Lin MY, Timmer FC, Oriel BS, Zhou G et al. Vestibular evoked myogenic potentials (VEMP) can detect asymptomatic saccular hydrops. Laryngoscope. 2006;116(6):987-92.

2. Rauch SD. Vestibular evoked myogenic potentials. Curr Opin Otolaryngol Head Neck Surg. 2006;14(5):299-304.

3. Ferber-Viart C, Duclaux R, Colleaux B, Dubreuil C. Myogenic vestibular-evoked potentials in normal subjects: a comparison between responses obtained from sternomastoid and trapezius muscles. Acta Otolaryngol. 1997;117(4):472-81.

4. Todd NP, Cody FW, Banks JR. A saccular origin of frequency tuning in myogenic vestibular evoked potentials? Implications for human responses to loud sounds. Hear Res. 2000;141(1-2):180-8.

5. Zhou G, Cox LC. Vestibular evoked myogenic potentials: history and overview. Am J Audiol. 2004;13(2):135-43.

6. McCue MP, Guinan Jr. JJ. Sound-evoked activity in primary afferent neurons of mammalian vestibular system. Am J Otol. 1997;18(3): $355-60$.

7. Welgampola MS, Colebatch JG. Characteristics and clinical applications of vestibular-evoked myogenic potentials. Neurology. 2005;64(10):1682-8.

8. Brantberg K, Tribukait A, Fransson PA. Vestibular evoked myogenic potentials in response to skull taps for patients with vestibular neuritis. J Vestib Res. 2003;13(2-3):121-30.

9. Cheng PW, Huang TW, Young YH. The influence of clicks versus short tone bursts on the vestibular evoked myogenic potentials. Ear Hear. 2003;24(3):195-7.

10. Huang TW, Cheng PW, Su HC. The influence of unilateral versus bilateral clicks on the vestibular-evoked myogenic potentials. Otol Neurotol. 2006;27(2):193-6.

11. Rauch SD, Merchant SN, Thedinger BA. Meniere's syndrome and endolymphatic hydrops. Double-blind temporal bone study. Ann Otol Rhinol Laryngol. 1989;98(11):873-83.

12. Bickford RG, Jacobson JL, Cody DT. Nature of average evoked potentials to sound and other stimuli in man. Ann N Y Acad Sci. 1964;112:204-23.

13. Cody DT, Jacobson JL, Walker JC, Bickford RG. Averaged evoked myogenic and cortical potentials to sound in man. Ann Otol Rhinol Laryngol 1964;73:763-77.

14. Townsend GL, Cody DT. The averaged inion response evoked by acoustic stimulation: its relation to the saccule. Ann Otol Rhinol Laryngol. 1971;80(1):121-31.

15. McCue MP, Guinan Jr. JJ. Influence of efferent stimulation on acoustically responsive vestibular afferent in the cat. J Neurosci. 1994;14(10):6071-83.

16. McCue MP, Guinan Jr. JJ. Acoustically responsive fibers in the vestibular nerve of the cat. J Neurosci. 1994;14(10):6058-70.

17. Jin Y, Nakamura M, Shinjo Y, Kaga K. Vestibular-evoked myogenic potentials in coclear implant children. Acta Otolaryngol. 2006;126(2):164-9.

18. Kelsch TA, Schaefer LA, Esquivel CR. Vestibular evoked myogenic potentials in young children: tests parameters and normative data. Laryngoscope. 2006;116(6):895-900.

19. Rosengren SM, Colebatch JG. Vestibular evoked potentials (VsEPs) in patients with severe to profound bilateral hearing loss. Clin Neurophysiol. 2006;117(5):1145-53.

20. Vanspauwen R, Wuyts FL, Van De Heyning PH. Validity of a new feedback method for the VEMP test. Acta Otolaryngol. 2006;126(8): 796-800.

21. Wit HP, Kingma CM. A simple model for the generation of the vestibular evoked myogenic potential (VEMP). Clin Neurophysiol. 2006;117(6):1354-8.

22. Wu Z, Zhang S et al. Significance of some otologic function tests in diagnosis of Meniere's disease. Lin Chuang Er Bi Yan Hou Ke Za Zhi. 2006;20(10):433-5.
23. Young YH. Vestibular evoked myogenic potentials: optimal stimulation and clinical application. J Biomed Sci. 2006.

24. Li MW, Houlden D, Tomlinson RD. Click evoked EMG responses in sternocleidomastoid muscles: characteristics in normal subjects. J Vestib Res. 1999;9(5):327-34.

25. Bath AP, Harris N, McEwan J, Yardley MP. Effect of conductive hearing loss on the vestibule-collic reflex. Clin Otolaryngol Allied Sci. 1999;24(3):181-3

26. Colebatch JG, Halmagyi GM, Skuse NF. Myogenic potentials generated by a click-evoked vestibulocollic reflex. J Neurol Neurosurg Psychiatry. 1994;57(2):190-7.

27. Robertson DD, Ireland DJ. Vestibular evoked myogenic potentials. J Otolaryngol. 1995;24(1):3-8.

28. Timmer FC, Zhou G, Guinan JJ, Kujawa SG et al. Vestibular evoked myogenic potentials (VEMP) in patients with Meniere's disease with drop attacks. Laryngoscope. 2006;116(5):776-9.

29. Murofushi T, Matsuzaki M, Wu CH. Short tone burst-evoked myogenic potentials on the sternoclideomastoid muscle: are these potentials also of vestibular origin? Arch Otolaryngol Head Neck Surg. 1999;125(6):660-4.

30. Rauch SD, Zhou G, Kujawa SG, Guinan JJ, Herrmann BS. Vestibular evoked myogenic potentials show altered tuning in patients with Meniere's disease. Otol Neurotol. 2004;25(3):333-8.

31. Halmagyi GM, Yavor RA, Colebatch JG. Tapping the head activates the vestibular system: a new use for the clinical reflex hammer. Neurology. 1995;45(10):1927-9.

32. Halmagyi GM, Aw ST, Karlberg M et al. Inferior vestibular neuritis. Ann N Y Acad Sci. 2002;956:306-13.

33. Halmagyi GM, Colebatch JG. Vestibular evoked myogenic potentials in the sternoclideomastoid muscle are not of lateral canal origin. Acta Otolaryngol. 1995;520 Suppl 1:1-3.

34. Baloh RW. Clinical practice. Vestibular neuritis. N Engl J Med. 2003;348(11):1027-32.

35. Goebel JA, O'Mara W, Gianoli G. Anatomic considerations in vestibular neuritis. Otol Neurotol. 2001;22(4):512-8.

36. Murofushi T, Halmagyi GM, Yavor RA, Colebatch JG. Absent vestibular evoked myogenic potentials in vestibular neurolabyrinthitis. An indicator of inferior vestibular nerve involvement? Arch Otolaryngol Head Neck Surg. 1996;122(8):845-8.

37. Minor LB, Cremer PD, Carey JP et al. Symptoms and signs in superior canal dehiscence syndrome. Ann N Y Acad Sci. 2001;942:259-73.

38. Merchant SN, Rosowski JJ, McKenna MJ. Superior semicircular canal dehiscence mimicking otosclerotic hearing loss. Adv Otorhinolaryngol. 2007;65:137-45.

39. Mikulec AA, McKenna MJ, Ramsey MJ et al. Superior semicircular canal dehiscence presenting as conductive hearing loss without vertigo. Otol Neurotol. 2004;25(2):121-9.

40. Rosowski JJ, Songer JE, Nakajima HH et al. Clinical experimental and theoretical investigations of the effect of superior semicircular canal dehiscence on hearing mechanisms. Otol Neurotol. 2004;25(3): 323-32.

41. Brantberg K, Bergenius J, Tribukait A. Vestibular evoked myogenic potentials in patients with dehiscence of the superior semicircular canal. Acta Otolaryngol. 1999;119(6):633-40.

42. Brantberg K, Bergenius J, Mendel L et al. Symptoms findings and treatment in patients with dehiscence of the superior semicircular canal. Acta Otolaryngol. 2001;121(1):68-75.

43. Murofushi T, Matsuzaki M, Mizuno M. Vestibular evoked myogenic potentials in patients with acoustic neuromas. Arch Otolaryngol Head Neck Surg. 1998;124(5):509-12.

44. Takeichi N, Sakamoto T, Fukuda S et al. Vestibular evoked myogenic potentials (VEMP) in patients with acoustic neuromas. Auris Nasus Larynx. 2001;28 Suppl:S39-41.

45. Kitamura K, Schuknecht HF, Kimura RS. Cochlear hydrops in association with collapsed saccule an ductus reuniens. Ann Otol Rhinol Laryngol. 1982;91 Part 1:5-13. 
46. Schuknecht HF. Pathophysiology of endolymphatic hydrops. Arch Otorhinolaryngol. 1976;212(4):253-62.

47. Schuknecht HF. Classification of endolymphatic hydrops. Am J Otol. 1984;5(6):528.

48. Schuknecht HF. The pathophysiology of Meniere's disease. Am J Otol. 1984; 5(6):526-7.

49. Schuknecht HF, Gulya AJ. Endolymphatic hydrops. An overview and classification. Ann Otol Rhinol Laryngol. 1983;106 Suppl:1-20.

50. Schuknecht HF, Richter E. Apical lesions of the cochlea in idiopathic endolymphatic hydrops and other disorders: pathophysiological implications. ORL J Otorrhinolaryngol Relat Spec. 1980;42(1-2):46-76.

51. Merchant SN, Adams JC, Nadol Jr. JB. Pathophysiology of Meniere's syndrome: are the symptoms caused by endolymphatic hydrops? Otol Neurotol. 2005;26(1):74-81.

52. Murofushi T, Shimizu K, Takegoshi H. Diagnostic value of prolonged latencies in the vestibular evoked myogenic potential. Arch Otolaryngol Head Neck Surg. 2001;127(9):1069-72.
53. De Waele C, Huy PT, Diard JP et al. Saccular dysfunction in Meniere's disease. Am J Otol. 1999;20(2):223-32.

54. Murofushi T, Matsuzaki M, Takegoshi H. Glycerol affects vestibular evoked myogenic potentials in Meniere's disease. Auris Nasus Larynx. 2001;28(3):205-8.

55. Shojaku H, Takemori S, Kobayashi $\mathrm{K}$ et al. Clinical usefulness of glycerol vestibular evoked myogenic potentials: preliminary report. Acta Otolaryngol. 2001;545 Suppl:65-8.

56. Modugno GC, Magnani G, Brandolini $C$ et al. Could vestibular evoked myogenic potentials (VEMPs) also be useful in the diagnosis of perilymphatic fistula? Eur Arch Otorhinolaryngol. 2006;263(6):552-5.

57. Carey JP. Intratympanic gentamicin for the treatment of Meniere's disease and other forms of peripheral vertigo. Otolaryngol Clin North Am. 2004;37(5):1075-90.

58. De Waele C, Menguenni R, Freyess G et al. Intratympanic gentamicin injections for Meniere's disease: vestibular hair cell impairment and regeneration. Neurology. 2002;59(9):1442-4. 